\title{
Exploratory pressure influences haptic shape perception via force signals
}

\author{
LuKas KaIM AND KNUT DREWING \\ University of Giessen, Giessen, Germany
}

\begin{abstract}
In this study of the haptic perception of small bumps, we investigated the influence of exploratory movement variation on signal integration and the percept's reliability. When sliding across a bump on a surface, the finger follows the geometry of the bump (i.e., the position signal). At the same time, patterns of forces depending on the gradient of the bump act on the finger (i.e., the force signal; Robles-de-la-Torre \& Hayward, 2001). Consistent with the maximum likelihood estimation (MLE) model, haptically perceived shape can be described by a weighted average of the shapes signaled by the position and force signals (Drewing \& Ernst, 2006; Ernst \& Banks, 2002). Here, we found that the weights of the position and force signals and the reliability of the shape percept depend on the pressure (and velocity) of the exploratory movement (Experiment 1). These effects could not be traced back to pressure effects on the reliability of the single signals, as would be predicted from the MLE model (Experiment 2). However, we found that the signal-specific shape estimate derived from the force signal increases with increasing pressure (Experiment 3), and this bias can explain the perceptual effects of exploratory pressure.
\end{abstract}

Haptic perception is inherently active (Gibson, 1962; Katz, 1969). In order to judge a haptic property, an observer has to touch and actively explore the object of interest with his or her hand. During the exploration, sensory signals are generated, from which the percept is then derived. Thus, haptic perception essentially depends on the executed exploratory movements. Recent studies (Drewing \& Ernst, 2006; Drewing, Wiecki, \& Ernst, 2008) have led to the suggestion that the principles of optimal integration of sensory signals that are known from passive perception also extend to active touch. In addition, the data hint at the possibility that individual variations in exploratory pressure and velocity might modulate signal integration (Drewing \& Ernst, 2006). Here, we investigated how and to what extent exploratory velocity and pressure influence signal integration and percept reliability, as well as whether these influences can be described by the known principles of optimal signal integration.

In recent years, the integration of redundant signals into a coherent percept has been studied extensively - in particular, the integration of different visual signals to depth, such as texture or disparity (Hillis, Watt, Landy, \& Banks, 2004), and the multimodal integration of, for example, seen and felt object size (Ernst \& Banks, 2002). Jacobs (2002) suggested that integration uses all signals available for a property. According to his model, signal-specific estimates $\hat{s}_{i}$ for the property are first derived from each signal $s_{i}$. Second, all estimates are combined into a coherent percept $\hat{s}$ by weighted averaging:

$$
\hat{s}=\sum_{i} w_{i} \hat{s}_{i},
$$

where

$$
\sum_{i} w_{i}=1 ; w_{i} \in[0,1] .
$$

The weighted average of signal-specific estimates describes the participants' percept of a property well when stimuli have been constructed to generate signals with conflicting information on this property (Backus, Banks, van Ee, \& Crowell, 1999; Young, Landy, \& Maloney, 1993). Furthermore, Landy, Maloney, Johnston, and Young (1995) predicted that averaging different estimates would increase the reliability $R_{\hat{s}}$ of the combined percept (which is the inverse of perceptual variance, $1 / \sigma_{\hat{s}}^{2}$ ); the lower discrimination thresholds for a property in multisignal than in singlesignal situations confirm this prediction (Perotti, Todd, Lappin, \& Phillips, 1998). It has also been suggested that the weights of signal estimates shift with the estimates' reliability (reliability-dependent weighting). Such a shift has been observed when external noise added to the display of one signal lowered both the reliability of that estimate and its weight (Backus et al., 1999; Young et al., 1993). Whereas most authors agree that an estimate's weight depends on its reliability, the circumstances that allow for weight choices that optimize the overall percept are still a topic of discussion (Rosas, Wagemans, Ernst, \& Wichmann, 2005). According to the maximum likelihood estimation (MLE) model, the reliability $R_{\hat{s}}$ of a percept is highest and the weights $w_{i}$ are optimal if the weights are proportional to the reliability of the signal estimates $R_{i}=1 / \sigma_{i}^{2}$ :

$$
w_{j}=\frac{R_{j}}{\sum_{i=1 \ldots, j, \ldots N} R_{i}} \text {, with } R_{\hat{s}}=\sum_{i} R_{i} \text {. }
$$


These equations hold when the noise distributions of the estimates are Gaussian and uncorrelated. If signals are processed by such distinct modalities as (for example) vision and touch, the estimates are likely to be uncorrelated. However, if different signals are processed by a single modality, they may share sources of noise. For example, different visual signals to depth are all based on the same retinal image. Oruç, Maloney, and Landy (2003) described in detail optimal integration for correlated estimates. In any case, both optimal (Alais \& Burr, 2004; Ernst \& Banks, 2002; Hillis et al., 2004) and suboptimal (Gepshtein, Burge, Ernst, \& Banks, 2005; Rosas et al., 2005) reliability-dependent weightings have been observed.

In haptic perception as well, observers use and combine multiple redundant signals (Srinivasan \& LaMotte, 1995; Voisin, Lamarre, \& Chapman, 2002). Robles-de-la-Torre and Hayward (2001) distiguished between the force and position signals for haptic shape perception: When sliding across a bump on a surface, the finger follows the geometry of the bump (i.e., the finger rises and lowers). This variation in the finger position is defined as the position signal. Simultaneously, the finger is exposed to patterns of forces whose directions depend on the slope of the surface (reaction forces from the object that are normal to the surface). Changes in force direction during exploration perturb the finger in temporal aspects of its movement (velocity, acceleration) along the surface geometry. The pattern of force directions thus creates a force signal to shape, in addition to the position signal. Note that both the force signal and the position signal alone provide information that would allow for full recovery of the shape. For natural shapes, the reaction forces are always normal to the surface and directly depend on a shape's geometry. Therefore, the force and position signals normally do correspond. Using a custom-made device, Robles-de-la-Torre and Hayward dissociated the force and position signals of small-scale bumps and holes. The device provided participants with the position signal of a plane, a hole, or a bump (3-mm amplitude) and, simultaneously, with force signals that conflicted with a position signal (e.g., position signal of a bump + force signal of a hole). For a stimulus with the position signal of a bump and the force signal of a plane, for example, the finger would stay in equilibrium on the slope regardless of how much a participant pushed on it, just as if the participant were exploring a flat surface. In that study, participants predominantly classified the presented shapes (bumps or holes) on the basis of the force signals. For example, they judged the physical geometry of a bump as a hole if the geometry of a bump (position signal) was perceived simultaneously with the force pattern (force signal) of a hole.

Drewing et al. (2008; Drewing \& Ernst, 2006) extended this research. Using a force feedback device, they decoupled the force and position signals to the perception of curvature of virtual 3-D arches: The position signal indicated one curvature of the arch, whereas the force signal indicated another. Participants compared such "inconsistent" arches with arches in which the signals were consistent with respect to curvature. The researchers showed that both signals, force and position, contributed to the perceived curvature, and that the curvature of a stimulus with inconsistent signals could be predicted from a weighted average of the curvatures indicated by the position and force signals. Furthermore, the signals' weights systematically shifted with variables that modified the signals' reliabilities. Both weighted averaging and reliabilitydependent weighting are consistent with the principles of signal integration postulated by the MLE model.

In their study, Drewing and Ernst (2006) observed pronounced interindividual differences in exploratory velocity ( $\sim 0.1$ to $0.27 \mathrm{~m} / \mathrm{sec})$ and executed pressure $(\sim 1$ to $3 \mathrm{~N})$. These differences correlated with pronounced interindividual differences in signal weights $(28 \%-64 \%$ force-signal weight; note that the position-signal weight equals $100 \%$ minus the force-signal weight). The forcesignal weight was higher at a higher pressure than at a lower pressure, as well as being lower at a higher velocity than at a lower velocity. The researchers speculated that variations in movement parameters might have directly influenced the single-signal reliabilities - and thus, indirectly, the signal weights - via reliability-dependent weighting. This speculation was the initial motivation for the present study.

An influence of movement variation on the percept reliability has been previously reported by Lederman and colleagues (Klatzky, Lederman, \& Reed, 1989; Lederman \& Klatzky, 1987). They demonstrated that observers habitually execute different exploratory procedures (EPs) to perceive different haptic properties of an object (e.g., exploratory pressure for softness, or contour following for shape) and that the EPs that participants use spontaneously are superior to other EPs in sensing the haptic dimension of interest. Lederman and colleagues speculated that EPs are executed in ways that even optimize a perception. An important precondition for such active movement optimization is that parametric movement variation within EPs systematically influences percept reliability. However, only a few studies have provided hints that variation in exploratory pressure might influence discrimination performance during softness perception (Srinivasan \& LaMotte, 1995) or perception of the small details of virtual stimuli (O'Malley \& Goldfarb, 2002).

Here, we investigated in detail the influence of movement variation on signal integration and percept reliability in the haptic perception of small bumps. In Experiment 1, we quantitatively investigated whether and to what extent the force- and position-signal weights and a percept's reliability indeed vary with variations in exploratory pressure and velocity. In Experiment 2, we examined whether the signal weight variations that we had observed with varying exploratory pressure could be explained by variations in the single-signal reliabilities. The results from this experiment suggested that shifts in the signal weights were not due to reliability shifts, but rather to biases in signalspecific estimates derived from the force signal. We designed Experiment 3 to test this hypothesis.

\section{EXPERIMENT 1}

We created stimuli using a PHANToM force feedback device. The device simulates haptic objects by monitoring 
the 3-D finger position and applying appropriate reaction forces to the finger. That is, if the finger indents a virtual surface, a reaction force is calculated (and generated) that drives the finger out of the object. In the standard rendering method, the magnitude of the reaction force linearly increases with the indentation depth, and force direction is normal to the surface. Here, we disentangled the position and force signals to Gaussian-shaped bumps (amplitudes varied between 1.5 and $4.5 \mathrm{~mm}, \sigma=7.5 \mathrm{~mm}$ ) on an otherwise planar surface. The geometry of the virtual bump determined the finger trajectory (i.e., the finger position during the stroke) - and thus the position signalvia the magnitude of the reaction forces. During exploration, the finger was deflected (i.e., was lifted or lowered) by reaction forces that created the same trajectory as if the finger explored a natural bump. However, the directions of the reaction forces, which specify the force signal for a shape, were taken from some other bump and were orthogonally projected onto the geometry (Figure 1). Note that the position and force signals could thus be independently manipulated and were independent in their effects on the finger, the position signal determining the shape of the trajectory and the force signal determining the velocity and acceleration of the movement along this shape. Furthermore, we enhanced the rendering method to create "quasi-hard" stimuli: Finger trajectories across these stimuli hardly varied with the manner of exploration (see the Results section for this experiment and the Appendix).

We constructed three standard bumps. Two of them had position and force signals that were inconsistent in terms of amplitude; that is, the amplitude indicated by the position signal differed from that indicated by the force signal. The third standard had consistent signals and served as a control stimulus. Participants always compared the perceived amplitude of one of the standards with the perceived amplitude of a bump with consistent signals (comparison). In each trial, the participants explored a standard and a comparison with a single stroke of the finger and decided which one was higher. We combined this two-alternative forced choice task with the method of constant stimuli. We varied the pressure (range: $1-2.2 \mathrm{~N}$ ) and the velocity (range: $0.1-0.22 \mathrm{~m} / \mathrm{sec}$ ) of the exploratory movement. These target pressures and velocities were chosen on the basis of measures of spontaneous behavior found in previous studies of shape discrimination (1-3 N, 0.10-0.27 m/ $\mathrm{sec}$ in Drewing \& Ernst, 2006; 0.16-0.25 m/sec in Voisin et al., 2002). We used $84 \%$ discrimination thresholds (just noticeable differences, or JNDs) as a measure of the percept's reliability, and points of subjective equality (PSEs) as a measure of the perceived bump amplitude. From the PSEs of the standards with inconsistent signals, we further calculated the relative force- and position-signal weights $\left(w_{\mathrm{F}}\right.$ and $\left.w_{\mathrm{P}}\right)$. We expected to confirm the correlative findings of Drewing and Ernst, which showed that the forcesignal weight increases with increasing pressure and decreases with increasing velocity.

\section{Method}

Participants. Eight participants ( 2 females, 6 males, age range 22-37 years, average 26 years; 7 right-handed, 1 left-handed) were paid for participating. None of them reported sensory or motor impairments of the index finger used in the experiment, and all had normal or corrected-to-normal vision and were naive to the purpose of the task.

A

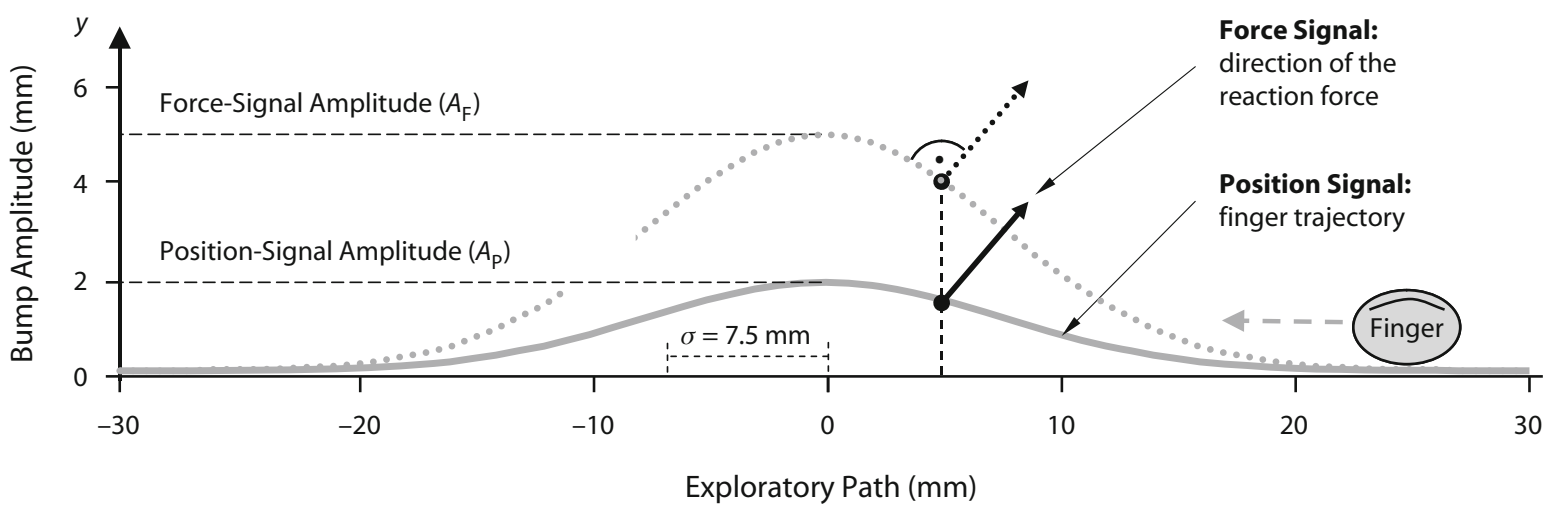

B

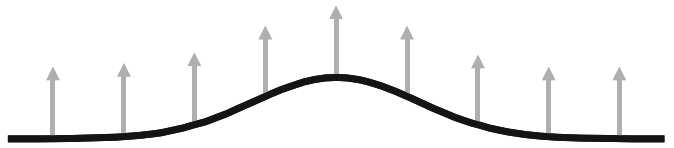

C

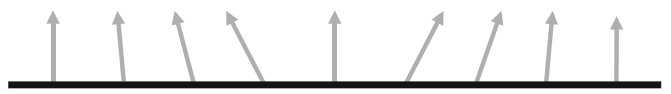

Figure 1. (A) We decoupled force from position signals using two independent Gaussian-shaped bumps, which only differed in their amplitude. One bump (dotted line) was used to define the directions of the reaction forces (force signal), which were then projected onto the geometry (and thus the position signal) of a bump with another amplitude (solid line). (B) Example of a "bump" with a force-signal amplitude of $A_{\mathrm{F}}=0$ and a position-signal amplitude $\boldsymbol{A}_{\mathrm{P}}>0$. During exploration of such a bump, the finger position varies (i.e., the finger rises and lowers), but there is no deceleration or acceleration of the finger along its trajectory. (C) Bump with $A_{\mathrm{P}}=0$ and $\boldsymbol{A}_{\mathrm{F}}>\mathbf{0}$. Force patterns decelerate and accelerate the finger while it moves across a planar surface. 
Apparatus. Observers sat in front of a custom-made visuohaptic workbench (Figure 2) comprising a PHANToM 1.5A haptic force feedback device $(1000 \mathrm{~Hz}, 0.03-\mathrm{mm}$ resolution, force in three translatory directions) and a 22-in. computer screen (Iiyama MA203 DT, $120 \mathrm{~Hz}, 1,280 \times 1,024$ pixels) connected to a PC. The index finger of the dominant hand was connected to the PHANToM via a thimblelike holder, which allowed for free finger movements with all six degrees of freedom in a workspace of about $42 \times 20 \times 18 \mathrm{~cm}^{3}$. Simultaneously, the participants, held still by a chinrest, looked onto the screen via stereo glasses (CrystallEyes) and a mirror $(40-\mathrm{cm}$ viewing distance). The mirror prevented the observers from seeing their hand and enabled spatial alignment of 3-D visuals with the haptic display. Custom-made software controlled the experiment, collected responses, and recorded the movements of the finger (position and reaction force; temporal resolution was $10 \mathrm{msec}$ ).

Stimuli. The PHANToM simulates haptic objects by applying reaction forces $\vec{F}_{j}$ to the finger in response to the 3-D finger position $j$. In the standard rendering model, force magnitude linearly increases with the indentation depth of the finger into a virtual object $\left(i_{j}\right.$, here the $y$-position of the surface in Figure 1), and force direction is normal to the object's surface $\left(\vec{n}_{j}=\right.$ normal vector at the surface position $j$ of the virtual object; $D=$ spring coefficient, which defines the stiffness of the virtual shape):

$$
\vec{F}_{j}=\vec{n}_{j} \cdot\left|\vec{F}_{j}\right|,\left|\vec{F}_{j}\right|=i_{j} \cdot D .
$$

Objects rendered with the standard method are of limited stiffness, and the shape of the finger trajectory across such objects depends on the applied pressure. To display "quasi-hard" shapes, we enhanced this rendering method in a way that keeps the indentation of the finger into the object constant during exploration. Note that in our experiments, participants moved their finger with prescribed pressure and velocity over two bumps on an otherwise planar surface. When the finger moved across the planar surface, indentation depth was already relatively constant, because the executed pressure was relatively constant. However, participants did not automatically follow the geometry of the bumps. Rather,

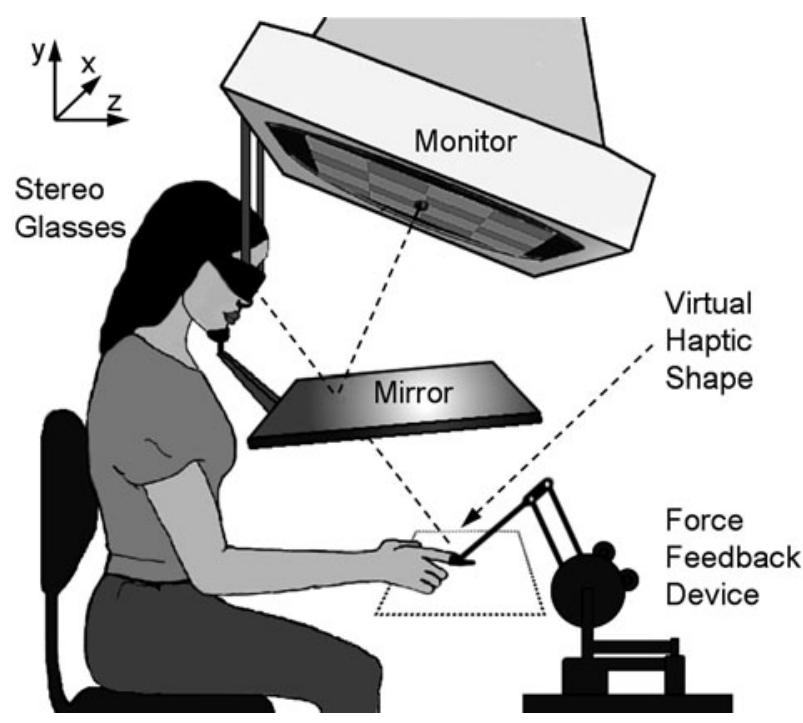

Figure 2. Visuohaptic setup. Participants explored the virtual shapes - displayed by a PHANToM force feedback device-with their index finger, connected to the device via a thimble-like holder. Simultaneously, they looked via stereo glasses and a mirror onto the screen. The mirror prevented participants from seeing their hand and enabled spatial alignment of a 3-D visual display with the haptic display. The PHANToM and screen were connected to a PC. Custom-made software controlled the experiment, collected responses, and recorded the movements of the finger. the finger pressed more strongly against a bump's surface when it "collided" with the bump (and was deflected upward) than when it passed the bump's peak (and was lowered). In this way, according to the standard rendering, the indentation depth - and thus the shape of the trajectory-slightly deviated from the target shape. This deviation would increase with increasing finger velocity and with a bump's increasing slope (i.e., slope variation in time). Our rendering method counteracts such deviation by taking into account the prescribed finger velocity $(V)$ and the slope of the bump at the current finger position $\left(m_{j}\right)$. To do this, we included a correction function $T_{\mathrm{C}}\left(V, m_{j}\right)$ in the calculation of the magnitude of the reaction force (i.e., Equation 4 below replaced the right part of Equation 3; for details, see the Appendix):

$$
\left|\vec{F}_{j}\right|=i \cdot D+T_{\mathrm{C}}\left(V, m_{j}\right) .
$$

This method rendered shapes across which the finger trajectory was almost constant for all bumps used and all manners of exploration (see the Results section below).

The bump shapes were defined by a modified Gaussian function ( $A=$ bump's amplitude, which varied; $\sigma=7.5 \mathrm{~mm}$ ):

$$
f(x)=A \cdot e^{-\frac{x^{2}}{2 \sigma^{2}}} .
$$

In each control cycle of the PHANToM, the actual finger position along the exploratory path ( $x$-position in Figure 1 ) received from the PHANToM was used to calculate (via Equation 5) the corresponding $y$-position $f(x)$ of the virtual bump. The difference between this value and the actual $y$-position of the finger is the indentation depth $i$ and was used to calculate the magnitude of the reaction force according to Equation 4. The spring coefficient $D$ was set to $2.5 \mathrm{~N} / \mathrm{mm}$.

To vary the position and force signals independently, we used two independent Gaussian functions that differed only in their amplitude (see Figure 1A): One function, defined by the amplitude $A_{\mathrm{P}}$ (position-signal amplitude), was used to define the shape of the finger trajectory (via the reaction force magnitude $\left|\vec{F}_{j}\right|$, Equation 4), and thus the position signal. The other function, defined by the force-signal amplitude $A_{\mathrm{F}}$, was used to calculate the directions of the reaction forces $\left(\vec{n}_{j}\right.$, left part of Equation 3$)$ from the first derivative of the Gaussian function. This function defined the force signal. If both amplitudes are equal, the reaction forces are normal to the surface. However, if, for example, $A_{\mathrm{F}}=0$ and $A_{\mathrm{P}}>0$ (Figure 1B), the force direction is always vertical and the forces determine only the shape of the trajectory. In contrast, if $A_{\mathrm{P}}=0$ and $A_{\mathrm{F}}>0$ (Figure 1C), the finger trajectory is flat but the force direction varies, so the finger is decelerated and accelerated, as would be caused by the slope of a bump.

The exploratory path was $15 \mathrm{~mm}$ (width) $\times 380 \mathrm{~mm}$ (length, along the $x$-axis) in the workspace (Figure 2). Bumps were defined along the path and extended over the entire width. The exploratory strokes started at the right side of the path, and the bumps' peak amplitudes were located at 150 and $310 \mathrm{~mm}$ distance from the path's start position (160 $\mathrm{mm}$ distance between the bumps' peaks).

Design. The design comprised three within-participants variables: pressure $($ lower $=1 \mathrm{~N}$, higher $=2.2 \mathrm{~N})$, velocity $($ lower $=$ $0.1 \mathrm{~m} / \mathrm{sec}$, higher $=0.22 \mathrm{~m} / \mathrm{sec}$ ), and standard bump (position-signal amplitude $A_{\mathrm{P}}[\mathrm{mm}] /$ force-signal amplitude $A_{\mathrm{F}}[\mathrm{mm}]=1.5 / 4.5 \mathrm{vs}$. $3 / 3$ vs. $4.5 / 1.5)$. The variables pressure and velocity combined to create four exploratory movement conditions. We measured the PSEs and JNDs of the standard bumps using the method of constant stimuli: Each standard was compared 16 times with each of the 13 comparison bumps. The comparisons had consistent position and force signals to the amplitude $\left(A_{\mathrm{P}}=A_{\mathrm{F}}=1.0-5.0 \mathrm{~mm}\right.$, in steps of $0.33 \mathrm{~mm}$ ).

The entire experiment consisted of eight sessions (45 min each, on 8 days within 1 month). In each session, all four movement conditions were presented block-wise and the order of blocks was balanced across sessions according to a Latin-square design. Within each movement condition, each stimulus pair (standard, comparison) was presented twice in randomized order (side of standard in 
exploratory path balanced). Thus, there were 4 (movement conditions) $\times 3$ (standards) $\times 13$ (comparisons) $\times 8$ (sessions) $\times 2$ (presentations) $=2,496$ trials per participant.

Procedure. At the beginning of each trial, a start scene was presented. Here, the finger position was displayed as a small sphere to guide participants toward the start position. Participants initiated a stimulus scene by pressing a start button. The sphere was hidden, and participants were instructed to make a single stroke along the exploratory path (from the right to the left side of the screen) with a prescribed pressure and velocity. Visual feedback supported participants in keeping these movement parameters at the prescribed values: Target velocity was displayed as a vertical line that moved repeatedly from the right to the left side of the screen, and target pressure was displayed as a fixed horizontal line in the middle of the screen. The current finger position and the executed pressure were correspondingly displayed as vertical and horizontal lines of different colors. The vertical "velocity" line represented the horizontal finger position, and the horizontal "pressure" line moved down with increasing pressure. The background was black. During the stroke, participants felt a standard and a comparison bump. While exploring the bumps, visual feedback was interrupted, to prevent a participant from receiving visual information about bump amplitude. After the stroke, participants decided whether the left or the right bump had been higher. All "buttonpresses" were done using the PHANToM. A trial was repeated later in the block when either the exploratory pressure or the velocity was not within predefined boundaries $( \pm 25 \%$ root-mean squared deviation within an area of $30 \mathrm{~mm}$ in front of each of the two bumps).

Prior to the experiment, participants were trained for about $45 \mathrm{~min}$ to execute exploratory movements with constant prescribed pressure and velocity (480 trials). In addition, they were informed that the individual with the "best perceptual performance" would be rewarded $(€ 20)$, which should have increased the participants' motivation to focus their attention mainly on the discrimination task.

Data analyses. We determined individual psychometric functions for each standard stimulus in each movement condition. That is, we plotted against the physical amplitude of the comparison the proportion of trials in which the comparison was perceived to be higher than the standard (see Figure 3 for a typical psychometric function).

The PSE is defined as the amplitude of the comparison stimulus at which discrimination performance is random (here, $50 \%$ ). The $84 \%$ discrimination threshold (JND) is defined as the difference between the PSE and the amplitude of the comparison when the comparison is judged to be higher than the standard $84 \%$ of the time. We obtained individual PSEs and JNDs by fitting cumulative Gaussians to the psychometric functions using the psignifit toolbox for MATLAB, which implements MLE methods (Wichmann \& Hill, 2001). The parameters $\mu$ and $\sigma$ of the cumulative Gaussian were used as estimates of PSE and JND, respectively. For the standard stimuli with inconsistent position and force signals (1.5/4.5 and 4.5/1.5), we calculated force-signal weights $\left(w_{\mathrm{F}}\right)$ from the individual PSEs (Drewing et al., 2008):

$$
w_{\mathrm{F}}=\frac{\mathrm{PSE}-A_{\mathrm{P}}}{A_{\mathrm{F}}-A_{\mathrm{P}}} .
$$

Note that the position-signal weight $\left(w_{\mathrm{P}}\right)$ is $1-w_{\mathrm{F}}$ (Equation 1). Finally, we analyzed finger position and reaction force data obtained from the PHANToM to check whether the executed pressure and velocity were within the predefined boundaries and whether the manipulation of the bump amplitude was successful (i.e., whether the measured trajectory amplitude matched the nominal one).

\section{Results}

PSEs. Individual PSEs for the standard stimulus with consistent signals (3.0/3.0) were entered into an ANOVA including the within-participants variables pressure and

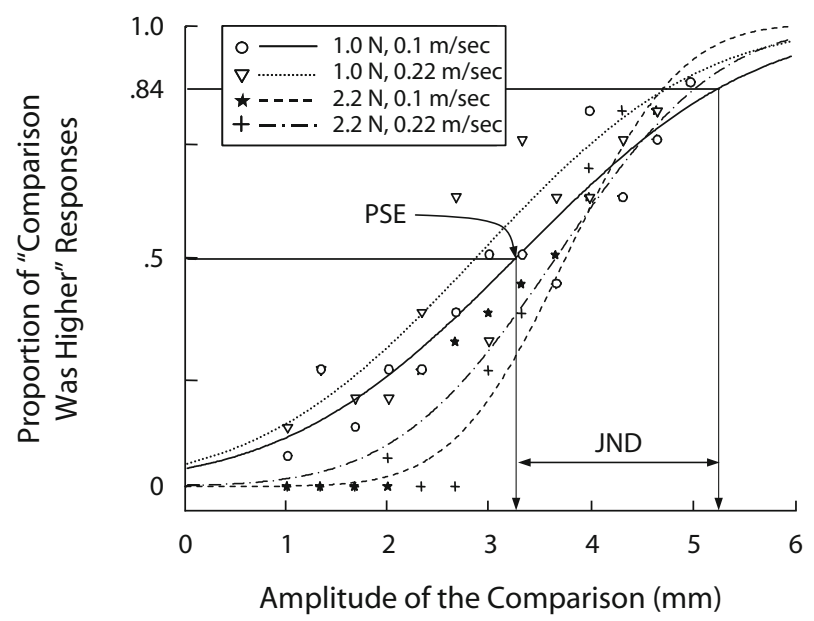

Figure 3. Typical individual psychometric functions, here obtained for the 1.5/4.5 standard $\left(A_{\mathrm{P}} / A_{\mathrm{F}}\right)$ in the four movement conditions in Experiment 1. The $x$-axis is the (physical) amplitude of the comparison stimuli, and the $y$-axis is the proportion of trials on which the comparison bump was perceived as being higher than the standard.

velocity. As should be the case, neither effect was significant $(p s>.05)$ and the average PSEs were close to the signals' true amplitudes (range, 2.97-3.10).

JNDs (Figure 4A). For the individual JNDs, an ANOVA on the variables pressure, velocity, and standard bump revealed a significant main effect of pressure $[F(1,7)=32.6, p<.005]$, indicating higher JNDs for the lower-pressure (1-N) than for the higher-pressure $(2.2-\mathrm{N})$ condition (1.46 vs. 1.18). Other effects were not significant $(p s>.12)$.

Weights (Figure 4B). Individual force-signal weights for standards with inconsistent position and force signals $\left(A_{\mathrm{P}} / A_{\mathrm{F}}=1.5 / 4.5\right.$ and $\left.4.5 / 1.5\right)$ were entered into an ANOVA including the within-participants variables pressure, velocity, and standard bump. A significant main effect of pressure $[F(1,7)=283.4, p<.001]$ indicated a considerably higher force-signal weight for the higher-pressure than for the lower-pressure condition (weight $=0.59$ vs. 0.37 , respectively). A significant main effect of standard bump $[F(1,7)=61.0, p<.001]$ indicated that the forcesignal weight was higher for the 1.5/4.5 standard than for the $4.5 / 1.5$ standard (0.59 vs. 0.37). A significant effect of velocity $[F(1,7)=23.8, p<.05]$ suggested a lower forcesignal weight at the higher velocity $(0.22 \mathrm{~m} / \mathrm{sec})$ than at the lower velocity $(0.1 \mathrm{~m} / \mathrm{sec})$. Other effects were not significant $(p \mathrm{~s}>.09)$.

Manipulation check: Executed pressure and velocity (Figure 5). For each successful trial, we calculated mean pressure and mean velocity in an area from 60 to $30 \mathrm{~mm}$ in front of each bump's peak. Overall, average scores did not differ by more than $10 \%$ from the prescribed values, which indicates that the participants maintained pressure and velocity quite well within the prescribed ranges. Individual averages were entered into ANOVAs comprising the variables pressure and velocity. For the actual pressure values, a main effect of pressure 
A

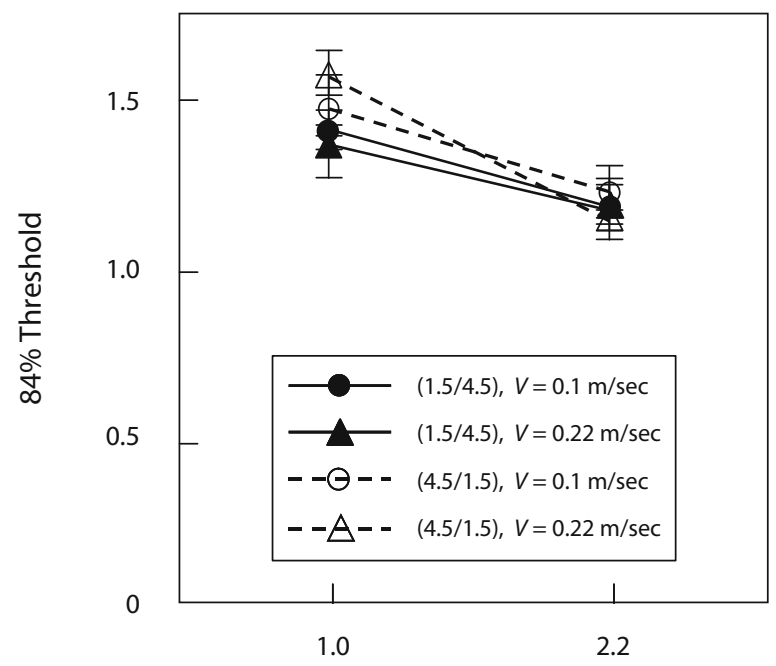

Pressure (N)
B

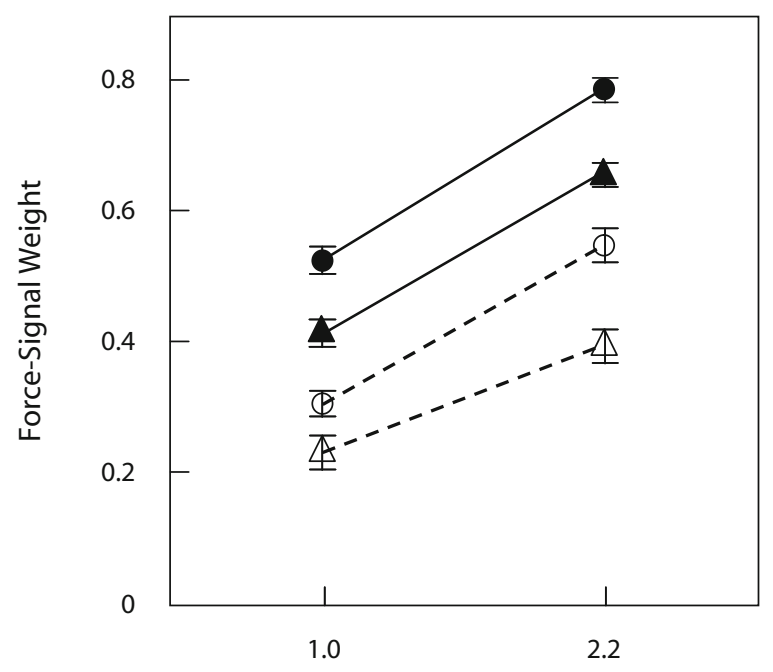

Pressure (N)

Figure 4. Experiment 1. Mean JNDs (A) and force-signal weights (B). Error bars are standard errors.

$[F(1,7)=1,631.1, p<.001]$ confirmed that the pressure manipulation was successful. For the actual velocities, a main effect of velocity $[F(1,7)=2,092.6, p<.001]$ confirmed that the velocity manipulation was successful. However, in the velocity data, a significant effect of pressure $[F(1,7)=18.5, p<.005]$ and an interaction between the variables pressure and velocity $[F(1,7)=6.0, p<.05]$ indicated that the higher-velocity movements (prescribed value $=0.22 \mathrm{~m} / \mathrm{sec}$ ) were executed slightly faster when combined with high pressure than when combined with low pressure (actual values $=0.216$ vs. $0.208 \mathrm{~m} / \mathrm{sec}$ for higher velocity, 0.102 vs. 0.100 for lower velocity). Other effects were not significant $(p \mathrm{~s}>.5)$.

Manipulation check: Bump amplitudes. We determined, trial by trial, the maximum amplitude $\Delta y_{\max }$ of the finger trajectory while exploring each stimulus. In an ideal case, $\Delta y_{\max }$ should equal the nominal amplitude $A_{\mathrm{P}}$ that defines the position-signal bump:

$$
\Delta y_{\max }=y_{\max }-\frac{y_{\operatorname{minR}}+y_{\min \mathrm{L}}}{2} \text {. }
$$

$y_{\max }$ is the maximum $y$-position of the finger during the exploration of a bump. $y_{\min \mathrm{L}}$ and $y_{\min \mathrm{R}}$ are the minima on the left and the right sides of the bump and were determined in an area of $\pm 30 \mathrm{~mm}$ around the bumps' nominal peak amplitudes. We calculated individual averages and standard deviations (between trials) of actual amplitudes for each stimulus under each movement condition. Averaged amplitudes ranged, over conditions, from $94 \%$ to $104 \%$ of the nominal ones, and averaged standard deviations did not exceed $0.15 \mathrm{~mm}$ (range $0.1-0.14 \mathrm{~mm}$, or in percentage of specified bump height, $3.0 \%-6.3 \%$ ) in either condition. These deviations of actual from nominal bump amplitudes are minor and will be ignored in further considerations.

\section{Discussion}

We varied the exploratory movement parameters pressure and velocity and then measured the contributions of the force and position signals to perceived bump amplitude and the JNDs. In the following discussion, we will report the weight results in terms of the force-signal weights only, because the force- and position-signal weights sum to 1 . Our results confirmed our predictions: The force-signal weight was higher for the higherpressure than for the lower-pressure condition and was lower for higher velocity than for lower velocity. Thus, we experimentally confirmed the correlative observation from Drewing and Ernst (2006) that the force-signal weight increases with increasing pressure and decreases with increasing velocity. Furthermore, the force-signal weights were always clearly greater than 0 and less than 1 , indicating that both force and position signals considerably contributed to perceived bump amplitude. Finally, JNDs were higher for the lower- than for the higher-pressure condition, indicating that the percept's reliability increased with increasing pressure.

Why did the weights shift with the exploratory movement parameters? According to the MLE model, estimate weights are proportional to the estimates' relative reliabilities (i.e., weights increase with reliability). In the present experiment, the force-signal weight was greater for the higher-pressure than for the lower-pressure conditions. One explanation for this weight increase could be that the force-signal reliability was higher for higher pressure. Because the reliability of the overall percept derives from the reliabilities of the force and position signals, the reliability of the overall percept should also be higher with higher pressure. But note that the forcesignal weight does not depend only on force-signal re- 


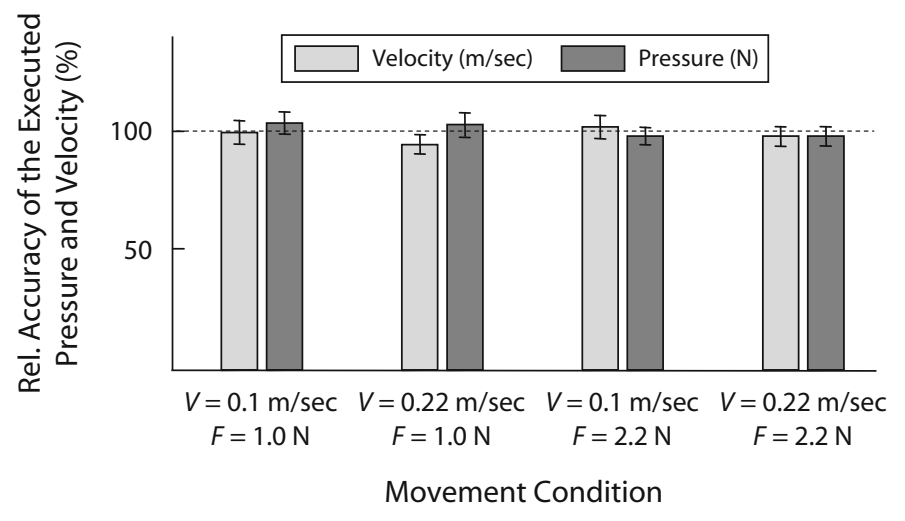

Figure 5. Experiment 1. Accuracy of the executed pressure and velocity in the four movement conditions relative to the prescribed values (in percentages; prescribed value $=\mathbf{1 0 0} \%$ ). Error bars are standard errors.

liability; it also - indirectly - depends on the positionsignal reliability, because the force- and position-signal weights sum to 1 . Thus, it could also be that the reliability of the position signal was lower (and accordingly the position-signal weight) with higher pressure, and that the force-signal weight was only indirectly influenced by the pressure variation. However, in that case, the percept's reliability should have been lower with higher pressure. In contrast, our results on JNDs indicated higher reliabilities with higher pressure. Thus, the observed shift in forcesignal weights and JNDs with pressure could be consistently explained within the framework of the MLE model, namely by the assumption that the force-signal - but not the position-signal-reliability increases with increasing pressure. We tested this explanation in Experiment 2.

The force-signal weight also varied between the two velocity conditions: It was lower for higher than for lower velocity. However, there was no variation in JNDs between the velocity conditions. Possibly, both signals were simultaneously and oppositely influenced by velocity. That is, the position-signal reliability might have decreased and the force-signal reliability increased with increasing velocity, and vice versa with decreasing velocity. Finally, our results revealed an interesting relationship between signal weights and signal amplitudes: The force-signal weight was higher for the $1.5 / 4.5$ standard $\left(A_{\mathrm{P}} / A_{\mathrm{F}}\right)$ than for the $4.5 / 1.5$ standard. This shift in signal weight could be explained by a reliability shift, if we assume that the forcesignal reliability is higher (or the position-signal reliability lower) for the $1.5 / 4.5$ than for the $4.5 / 1.5$ standard. This would mean, however, that a "force-signal threshold" (or "position-signal threshold") is lower for a signal amplitude of $4.5 \mathrm{~mm}$ than for $1.5 \mathrm{~mm}$; this contradicts Weber's law, which states that absolute thresholds increase proportional to the signal magnitude. Furthermore, there was no variation in the overall threshold between the two standards. Therefore, we suppose that the observed amplituderelated weight shift cannot be explained by a reliability shift. We will discuss this issue later in detail.

\section{EXPERIMENT 2}

In Experiment 2, we investigated whether the effects of exploratory pressure on signal weights and on the percept's reliability can be traced back to force-signal reliability, which could be concluded from applying the MLE model. Therefore, we measured JNDs (discrimination thresholds) for force-signal analogues without providing position signals and JNDs for position-signal analogues without providing force signals, and we varied the exploratory pressure $(1,1.6,2.2 \mathrm{~N})$. We did not vary velocity, mainly for practical reasons (i.e., to reduce the length of the experiment).

In active touch, it is problematic to present a force signal to a bump without presenting any position signal to that bump. Even when the finger slides across a planar surface, there is a constant position signal that contributes to the impression of a planar surface. A similar argument can be applied to presenting position without force signals. Hence, we decided to measure the JNDs of single-signal analogues using passive conditions. In passive touch, we instructed participants to discriminate between the amplitudes of two successive (passive) liftings of their finger rather than between two bumps - analogous to receiving a position signal only. Likewise, they had to discriminate between patterns of lateral forces applied to their fingeranalogous to receiving a force signal only. The finger liftings and lateral force patterns were identical to, respectively, the position and force signals of bumps moving beneath a stationary finger with constant velocity $(0.16 \mathrm{~m} /$ $\mathrm{sec}$ ). We used the obtained single-signal JNDs to predict both overall JNDs (JNDs of the overall percept) and forcesignal weights, for the case of optimal integration of force and position signals into a coherent bump percept (according to the MLE model, Equation 2). We tested these predictions in active conditions similar to those in Experiment 1, which included combinations of the force and position signals used in the passive conditions. Qualitatively, we predicted, on the basis of the MLE model and the results 
of Experiment 1, that the overall JNDs in the active conditions and the force-signal-only JNDs from the passive conditions would decrease with increasing pressure, whereas the force-signal weight would increase.

\section{Method}

Participants. Eight right-handed healthy male participants (2437 years old, average 28 years) were paid for participating; all were naive to the purpose of the experiment.

Apparatus and Stimuli. These were as in Experiment 1.

Design. We used passive conditions to separately measure discrimination thresholds (JNDs) for the position and force signals. The experimental design in passive conditions comprised two withinparticipants variables: pressure $($ low $=1 \mathrm{~N}$, middle $=1.6 \mathrm{~N}$, high $=$ $2.2 \mathrm{~N})$ and signal amplitude $\left(A_{\mathrm{P}} / A_{\mathrm{F}}=5 /-, 8 /-,-15\right.$, or $\left.-/ 8\right)$. We measured the JND of each standard stimulus with respect to a range of 13 comparisons. The amplitude analogues of the comparisons varied in steps of $0.5 \mathrm{~mm}$ from 2 to $8 \mathrm{~mm}$ for the $5-\mathrm{mm}$ standards, and from 5 to $11 \mathrm{~mm}$ for the 8 -mm standards.

In the active conditions, we combined the force- and position-signal amplitudes of the standards from the passive conditions into two standards with inconsistent signals $(5 / 8,8 / 5)$. The experimental design in the active conditions comprised two within-participants variables: pressure $($ low $=1 \mathrm{~N}$, middle $=1.6 \mathrm{~N}$, high $=2.2 \mathrm{~N})$ and standard bump (5/8 or $8 / 5)$. We measured the PSEs and JNDs of the standards with respect to a range of 13 comparisons with consistent signals, and the amplitude range was 3.5-9.5 $\mathrm{mm}$ (steps of $0.5 \mathrm{~mm}$ ).

The entire experiment consisted of eight sessions ( $1 \mathrm{~h}$ each, on 8 days within 7 weeks). In each session, both a passive and an active part were presented, and the sessions started alternately with an active or a passive part. The three pressure conditions were presented blockwise in a randomized order, and trials were randomized within blocks. All in all, there were 3 (pressure conditions) $\times 4$ (standards) $\times 13$ (comparisons) $\times 2$ (presentations) $\times 8$ (sessions $)=2,496$ trials per participant in the passive part, and 3 (pressure conditions) $\times 2$ (standards $) \times 13$ (comparisons) $\times 2$ (presentations $) \times 8$ (sessions $)=$ 1,248 trials per participant in the active part (16 repetitions per standard-comparison pairing in each pressure condition).

Procedure. In the passive conditions, after self-initiating a trial, participants were instructed to press down with a given constant force (visual feedback). After 1,200 msec (time to adjust the pressure), either the force or the position signals of two Gaussian bumps (standard and comparison) "moved beneath" the finger with a constant velocity of $0.16 \mathrm{~m} / \mathrm{sec}$. More precisely, to measure JNDs for position signals, in each trial participants discriminated between two successive liftings of their finger and were instructed to decide which finger deflection was larger. The two liftings were identical to the position signals of two bumps that moved with a constant velocity beneath the stationary finger. To measure JNDs for force signals, the participants discriminated between two lateral force profiles applied to the finger and decided which force was stronger. The force profiles were identical to the force signals of two bumps that moved beneath the finger. In the active conditions, the procedure was the same as in Experiment 1: In each trial, participants were instructed to actively explore a standard and a comparison stimulus within a single stroke and to decide whether the left or the right bump was higher (prescribed velocity: $0.16 \mathrm{~m} / \mathrm{sec}$ ).

Data analyses. These were as in Experiment 1. Additionally, we used the individual JNDs for force and position signals $\left(\sigma_{\mathrm{F}}, \sigma_{\mathrm{P}}\right)$ obtained from the passive conditions to predict individual overall JNDs $\left(\sigma_{\text {pred }}\right)$ according to the MLE model (Ernst \& Banks, 2002):

$$
\sigma_{\text {pred }}=\sqrt{\frac{\sigma_{\mathrm{P}}^{2} \sigma_{\mathrm{F}}^{2}}{\sigma_{\mathrm{P}}^{2}+\sigma_{\mathrm{F}}^{2}}} .
$$

We compared the means of these predicted overall JNDs with the mean measured overall JNDs. Next, as with the JNDs, we predicted the force-signal weights $\left(w_{\mathrm{F}(\text { pred })}\right)$ for the two inconsistent bumps (5/8 and $8 / 5)$ according to the MLE model:

$$
w_{\mathrm{F}(\text { pred })}=\frac{\sigma_{\mathrm{P}}^{2}}{\sigma_{\mathrm{P}}^{2}+\sigma_{\mathrm{F}}^{2}} .
$$

Then we compared the mean predicted with the mean measured force-signal weights.

\section{Results}

JNDs for force and position signals in single-signal conditions (Figure 6A). As predicted, JNDs for force signals systematically decreased with increasing pressure, whereas JNDs for position signals were not reliably influenced by the pressure level. JNDs for both force and position signals were higher for the 8-mm than for the 5-mm signal. Statistically, two ANOVAs (for the variables pressure and signal amplitude, conducted separately for force- and position-signal JNDs) revealed main effects of signal amplitude for both position-signal JNDs $[F(1,7)=$ 29.6, $p<.01]$ and force-signal JNDs $[F(1,7)=9.5, p<$ .05]. Furthermore, the ANOVAs revealed a main effect of pressure for force-signal JNDs $[F(2,14)=7.3, p<.01]$. Difference contrasts between successive pressure levels confirmed the predicted systematic increase of forcesignal JNDs with pressure [1 vs. $1.6 \mathrm{~N}, t(7)=2.59, p<$ $.05 ; 1.6$ vs. $2.2 \mathrm{~N}, t(7)=2.82, p<.05$, single-sided]. Other effects were not significant $(p \mathrm{~s}>.1)$.

Predicted and measured overall JNDs (Figure 6B). From the single-signal JNDs, we first predicted overall JNDs for the two standard bumps, 5/8 and 8/5 $\left(A_{\mathrm{P}}[\mathrm{mm}] /\right.$ $\left.A_{\mathrm{F}}[\mathrm{mm}]\right)$. An ANOVA on the predicted JNDs (variables: pressure and standard bump) revealed a significant main effect of pressure $[F(2,14)=11.2, p<.01]$ and a significant interaction. These results indicate that pressuredependent JND variation is predicted to differ between the two standard bumps. Hence, we analyzed predicted JNDs separately for the $5 / 8$ and $8 / 5$ standards using pairwise $t$ tests between successive pressure levels. For the $8 / 5$ standard, predicted JNDs were significantly higher for the pressure level of $1 \mathrm{~N}$ relative to $1.6 \mathrm{~N}[t(7)=4.84, p<$ $.01]$. Predictions for the pressure levels of 1.6 and $2.2 \mathrm{~N}$ did not reliably differ $(t<1)$. For the $5 / 8$ standard, neither of these two comparisons reached significance $(p s>.2)$.

In the next step, actual JNDs measured in the active conditions were submitted to the same analyses as the predicted JNDs. In contrast to the analysis for the predicted JNDs, this ANOVA revealed only that measured JNDs are lower for the $5 / 8$ than for the $8 / 5$ standard $[F(1,7)=$ $5.5, p<.05]$ (other effects, $p \mathrm{~s}>.10$ ). However, pairwise $t$ tests between successive pressure levels for each standard confirmed the predicted pattern of pressure effects: For the $8 / 5$ standard, measured JNDs were significantly higher for the pressure level of $1 \mathrm{~N}$ than for the level of $1.6 \mathrm{~N}[t(7)=2.18, p<.05]$, but they did not differ reliably between 1.6 and $2.2 \mathrm{~N}(t<1)$. For the $5 / 8$ standard, neither of these comparisons reached significance ( $p s>.2$, single-sided tests).

Predicted and measured force-signal weights (Figure 7A). Force-signal weights were also predicted 


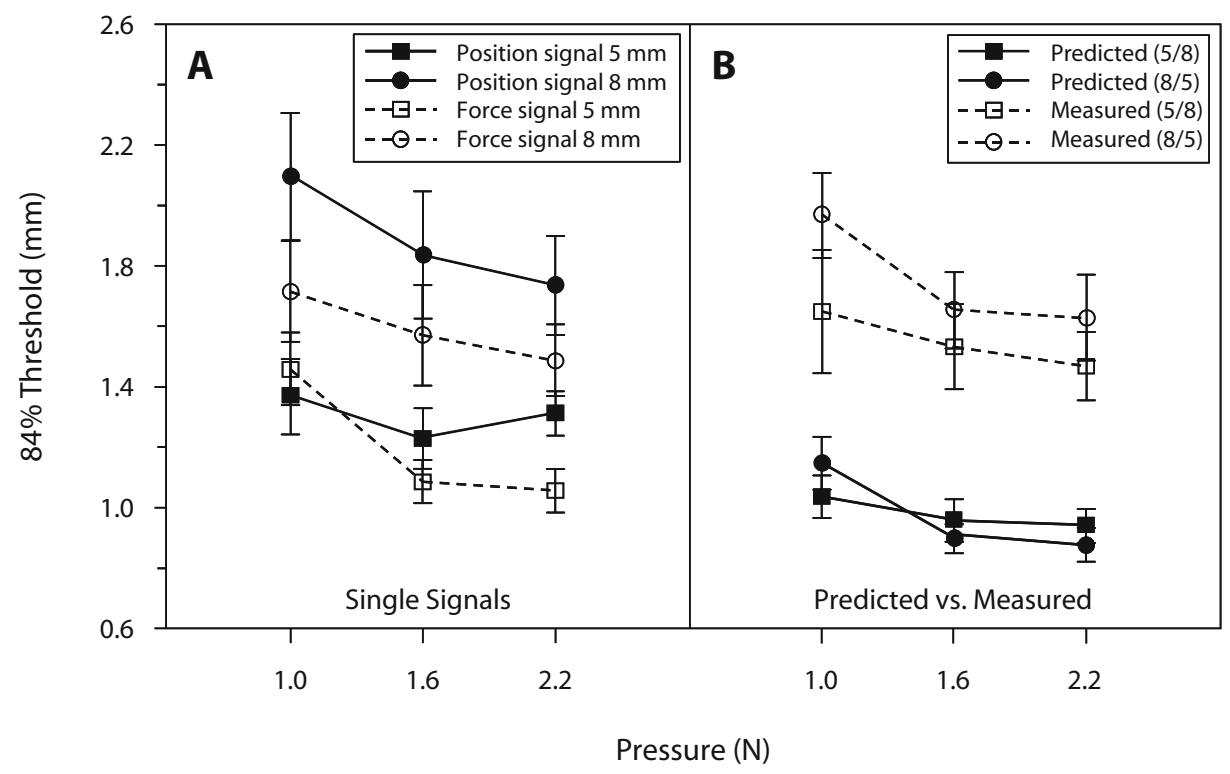

Figure 6. Experiment 2. (A) Single-signal JNDs measured in the passive conditions. Note that there was no significant effect of pressure on position-signal JNDs (solid lines, $p>$.1). (B) Predicted overall JNDs and overall JNDs measured in active conditions with both signals. Error bars are standard errors.

from the single-signal JNDs. An ANOVA (variables: pressure and standard bump) revealed higher predicted force-signal weights for the $8 / 5$ than for the $5 / 8$ standard $[F(1,7)=44.1, p<.001]$. Other effects were not significant $(p>.2)$. In contrast, the same analysis on the measured force-signal weights $\left(w_{\mathrm{F}(\text { meas }}\right)$, calculated from PSEs according to Equation 7) revealed a higher actual force-signal weight for the $5 / 8$ than for the $8 / 5$ standard $[F(1,7)=9.6, p<.05]$. It further indicated a systematic increase of force-signal weight with increasing pressurewhich was predicted from Experiment 1, but not from the single-signal JNDs [main effect in ANOVA, $F(2,14)=$ $6.5, p<.05$; difference contrasts: 1 vs. $1.6 \mathrm{~N}, t(7)=1.56$, $p=.08 ; 1.6$ vs. $2.2 \mathrm{~N}, t(7)=2.97, p<.01]$.

\section{Discussion}

As we had expected, in conditions including both force and position signals, the force-signal weight increased with increasing pressure, and the overall JNDs decreased-at least for one of the two standards. Also as expected, JNDs measured separately for the force signal decreased with increasing pressure, whereas JNDs for the position signal were not influenced by pressure. Qualitatively, this is consistent with our hypothesis that pressure effects on signal weights and on the overall percept's reliability (measured by overall JND) are due to pressure effects on force-signal reliability. However, this hypothesis was derived from the MLE model. Quantitatively, the model predicted the effects of pressure only on the JNDs, not on the weights. Hence, we conclude that the reliability of the overall percept shifted with force-signal reliability, but other factors must be responsible for the effects on the weights. We will now explain this finding in detail.
Predicted and measured overall JNDs. We used the single-signal JNDs measured in the passive conditions to predict - according to the MLE model of optimal signal integration - the overall discrimination JNDs for stimuli defined by both force and position signals $(5 / 8$ and $8 / 5)$. The pressure effects we observed corresponded to the predicted ones (and, by and large, to effects on the forcesignal JNDs; see Figure 6): Both predicted and measured JNDs were higher in the low-pressure than in the middlepressure conditions for the $8 / 5$ standard, whereas they did not differ reliably for the $5 / 8$ standard. Hence, we conclude that pressure effects on the overall JND are a function of pressure effects on the single-signal JNDs, and in particular on the force-signal JNDs. However, whereas the pattern of JND variation is consistent with the MLE predictions, the magnitude of the measured JNDs is not. The measured JNDs were considerably larger than predicted (on average, 1.75 vs. $0.98 \mathrm{~mm}$ for the $5 / 8$ standard and 1.56 vs. $0.99 \mathrm{~mm}$ for the $8 / 5$ standard). One may speculate that this discrepancy is due to correlations between the forceand position-signal estimates. That is, although both signals are defined to be physically independent, they might be partly processed by the same mechanoreceptors and share a source of noise. Our predictions were made under the assumption of uncorrelated estimates. Indeed, positively correlated estimates would benefit less from optimal integration than we had predicted (Oruç et al., 2003). However, optimal integration, also of correlated signals, cannot predict overall JNDs that are larger than the JNDs for one of the single signals (because selecting that signal would be the better strategy; Oruç et al., 2003). This, however, is just what we observed in our data (Figure 6). Thus, correlations between force- and position-signal es- 


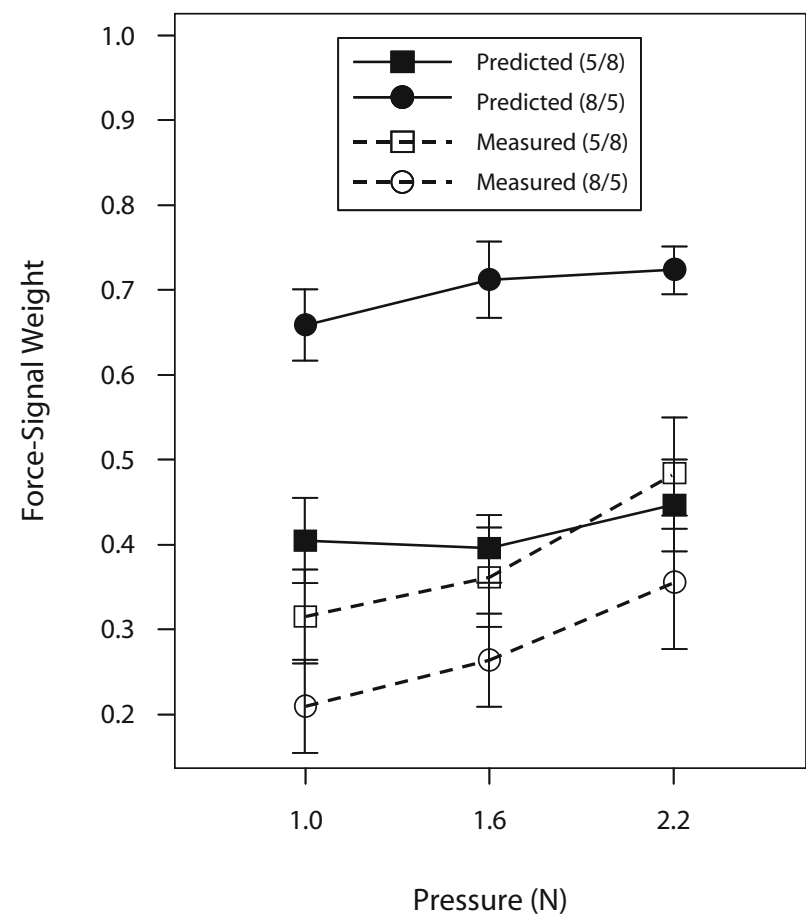

Figure 7. Experiment 2. Predicted (solid lines) and measured (dashed lines) force-signal weights. Error bars are standard errors.

timates can only partly explain the discrepancy between our measured and predicted JNDs. A further reason for the discrepancy could be the fact that we had to make predictions from passive conditions, which were then tested in active conditions: The active task may have been more difficult than the passive tasks, and hence, discrimination performance was impaired in the active conditions. The phenomenon called tactile suppression (Blakemore, Wolpert, \& Frith, 1998; Vitello, Ernst, \& Fritschi, 2006) may also have contributed to the discrepancy. According to this phenomenon, tactile sensitivity is reduced during voluntarily executed body movements.

Predicted versus measured force-signal weights. The magnitude of the measured signal weights $(0<$ $w<1$ ) demonstrated that both the force and position signals contribute to an integrated bump percept. However, measured force-signal weights showed hardly any similarity to the predicted weights. Note that we additionally tried to predict the measured weights under the assumption of positively correlated force- and position-signal estimates (see Equation 5 in Oruç et al., 2003). However, the best predictions (according to a least-squares criterion) were obtained assuming a correlation of 0 . So correlated estimates provide no explanation for the mismatch between the observed and predicted weights. In detail, we did not reliably predict an increase of force-signal weight with pressure, but we have observed such an increase in the measured weights (here and in Experiment 1). Second, the measured force-signal weights were clearly lower than predicted (on average, .34 vs. .57). Finally, we pre- dicted from the single-signal JNDs that the force-signal weight would be considerably higher for the $8 / 5$ (positionsignal-dominant) standard than for the 5/8 (force-signaldominant) standard. However, we found just the reverse: The force-signal weight was higher for the force-signaldominant than for the position-signal-dominant standard. Note that the standard with the higher force-signal weight is also the one for which the force signal indicated a higher amplitude than the position signal (and vice versa for the standard with the lower force-signal weight). This observation is consistent with the amplitude-related weight shift observed in Experiment 1. Taken together, these findings lead us to conclude that neither the effect of pressure nor of the standard on the force-signal weight can be explained by shifts in the single-signal reliabilities, as was predicted by the MLE model. Other factors must be responsible.

A recent study has reported several biases in amplitude estimates derived from the force signal (Drewing \& Kaim, 2009): Estimates derived from the same force-signal amplitudes had different values, depending on the exploratory conditions. Drewing and Kaim suggested that the brain can use only reduced aspects of the force signal rather than the entire signal (which would allow unbiased recovery of the shape). Can biases related to exploratory pressure also explain the weight effects in the present study? To answer this question, it should be remembered that the (measured) force-signal weights were obtained from the PSEs (Equation 6). Figure 8 shows that effects in the measured weights are due to a systematic increase of PSEs with increasing pressure for the force-signal-dominant stimulus (5/8) and a systematic decrease of PSEs for the position-signal-dominant stimulus (8/5). The PSEs were determined by comparisons of the standards with stimuli that had consistent signals to amplitude $\left(A_{\mathrm{P}}=A_{\mathrm{F}}\right)$. Now let us assume a pressure-related bias, namely that with higher pressure, estimates with larger amplitude are de-

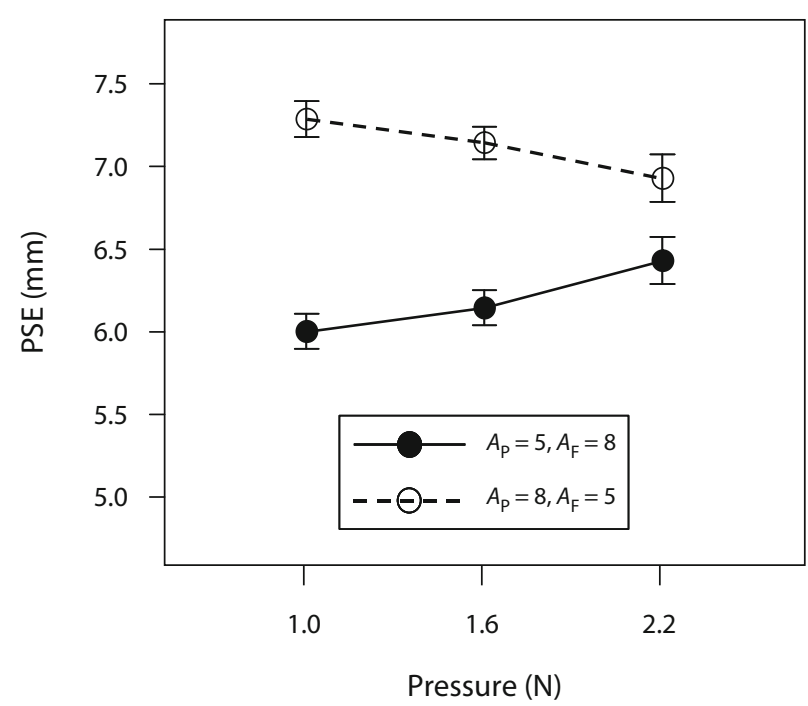

Figure 8. Experiment 2. Points of subjective equilibrium (PSEs) measured for the 5/8 standard (solid line) and the 8/5 standard (dashed line). Error bars are standard errors. 
rived from the same force signals. We explain the effect of this bias separately for each of the two standards: For the force-signal-dominant standard (5/8), the force-signal amplitude is larger for the standard than for the comparison that corresponds to the PSE (e.g., 8 vs. $5.96 \mathrm{~mm}$ for the 1-N condition; see Figure 8). Thus, the bias increases the estimate derived from this standard's large force-signal amplitude to a great extent, but it increases the estimate derived from the comparison's smaller force-signal amplitude to a lesser extent. As a consequence, the perceived amplitude for this standard increases more strongly with increasing pressure than the perceived amplitude of the comparisons. However, the comparison stimulus that corresponds to this standard - and thus, the PSE - must then itself be larger with higher pressure. This was indeed the case. For the position-signal-dominant standard (8/5), the situation is just the other way around: The force-signal amplitude is smaller for this standard than for the relevant comparison stimuli. Given that higher pressure results in greater amplitude estimates derived from the force signal, the perceived amplitude of this standard increases less with increasing pressure than the perceived amplitude of the comparison; thus, the PSE must be smaller-as was also the case here. Hence, the present findings on the measured signal weights could possibly be explained by a pressure-related bias on amplitude estimates derived from the force signal. Experiment 3 tests this explanation.

\section{EXPERIMENT 3}

The aim of Experiment 3 was to investigate whether the (absolute) signal-specific amplitude estimates derived from the force signal $\left(\hat{s}_{\mathrm{F}}\right)$, but not those from the position signal $\left(\hat{s}_{\mathrm{P}}\right)$ vary with variations in pressure (i.e., whether they are biased). In a matching task, participants compared actively explored haptic bumps with visually presented bumps in two pressure conditions ( 1 or $2.2 \mathrm{~N})$. We used three types (sets) of haptic stimuli: varying only $A_{\mathrm{P}}$ (so $A_{\mathrm{F}}=0$; position-signal bumps, Figure 1B) or $A_{\mathrm{F}}\left(A_{\mathrm{P}}=0\right.$; force-signal bumps, Figure $1 \mathrm{C}$ ), or simultaneously varying both amplitudes ( $A_{\mathrm{P}}=A_{\mathrm{F}}$; consistent bumps). Amplitudes varied between 2.0 and $5.5 \mathrm{~mm}$. The haptic stimuli of the three different types were presented in a randomized order, and participants always matched them with the same set of visual stimuli. Thus, participants matched the perceived amplitude of each explored stimulus on the same visual scale, independent of the experimental condition. We used the visually matched amplitudes as a measure of haptically perceived amplitude. For each stimulus type and pressure condition, we further calculated individual linear regressions of visually matched amplitudes on the haptic signal amplitudes. The regression intercepts did not differ between conditions (this lack of effect was expected from Drewing \& Kaim, 2009). Thus, we could use the slopes of the regressions to measure how absolutely amplitude increases were perceived with increasing signal amplitudes, as varied in each of the stimulus types.

As already mentioned in Experiment 2, it is difficult to measure signal-specific estimates directly, because it is difficult to strictly isolate the force signal to bumps from the position signal. In Experiment 3, we instead measured increases in absolutely perceived amplitude with separate increases in either of the signals' physical amplitude. This measure depends on both signal-specific amplitude estimates and estimate weighting. However, it did still allow us to test our assumption from Experiment 2-namely that pressure biases the force-signal estimate but affects neither the position-signal estimate nor the weighting of the estimates. Hence, we predict that the increase in perceived amplitude for position-signal bumps will not depend on pressure, because pressure should affect neither the position-signal estimate nor the estimates' weights. For the force-signal bumps, however, the increase in perceived amplitude should be larger with higher pressure, because of pressure-related bias in the force-signal estimate. Finally, for the consistent-signal bumps, we also expect a larger amplitude with increased pressure, again because of biased force-signal estimates.

\section{Method}

Participants. Eight right-handed healthy participants ( 1 female, 7 male; 23-37 years old, average 28 years) participated for pay.

Apparatus and Stimuli. The haptic stimuli and the apparatus were the same as in Experiments 1 and 2. The visual stimuli were displayed as Gaussian-shaped line drawings (line width $=1 \mathrm{~mm}$ ) on a dark background. The length $(60 \mathrm{~mm} \hat{=}[-4 \sigma: 4 \sigma])$ and amplitude (range $=2-5.5 \mathrm{~mm}$, in steps of $0.5 \mathrm{~mm}$ ) of visual bumps were the same as for the haptic bumps. For the matching task, the visual bumps were displayed simultaneously, grouped as a $2 \times 4$ array, in which the amplitude increased from the upper-left to the lower-right bump.

Design and Procedure. The experimental design comprised three within-participants variables: pressure (higher $=1 \mathrm{~N}$, lower $=$ $2.2 \mathrm{~N})$, amplitude $(2.0,2.5,3.0,3.5,4.0,4.5,5.0$, or $5.5 \mathrm{~mm})$, and stimulus type (force-signal, position-signal, or consistent bumps). There were three sets (types) of haptic stimuli. We either varied only $A_{\mathrm{P}}\left(A_{\mathrm{F}}=0\right)$ or $A_{\mathrm{F}}\left(A_{\mathrm{P}}=0\right)$ or simultaneously varied both amplitudes $\left(A_{\mathrm{P}}=A_{\mathrm{F}}\right)$. Each haptic stimulus was presented five times in each of the two pressure conditions. Stimuli of all three types were presented in a randomized order within each pressure condition. The pressure conditions were presented block-wise within one session, which started with either the $1-\mathrm{N}$ or the $2.2-\mathrm{N}$ condition (randomized). All in all, there were 240 ( 2 pressure conditions $\times 3$ stimulus types $\times 8$ stimuli $\times 5$ repetitions) trials per participant. In each trial, participants actively explored a single bump. After the exploratory stroke, the decision scene was presented, and participants matched the previously felt amplitude with one of the eight displayed visual bumps. The participants made decisions by moving the finger (displayed as a small spot) over one of the eight visual bumps.

Prior to the experiment, participants had trained to match haptic bumps with the corresponding visual bumps for about $15 \mathrm{~min}$ by exploring bumps with consistent signals. After an exploration, they obtained feedback about the visual bump that corresponded to the previously explored haptic bump.

Data analyses. For each single haptic stimulus and each pressure condition, we calculated the individual means of visually matched amplitude (five values/stimulus). We then calculated individual linear regressions of visually matched amplitudes (labeled here $\left.A_{\mathrm{V}}\right)$ on the haptic signal amplitudes $\left(A_{\mathrm{F}}\right.$ or $A_{\mathrm{P}}$, eight stimuli/ regression), for each stimulus type and pressure condition. Statistically, the linear regression model explained most of the variance in the majority of the individual data sets (median $r^{2}=.83 ; 1 \mathrm{st}$ quartile $=.74,3$ rd quartile $=.91$ ). Thus, the perceived amplitudes linearly increased with increasing haptic signal amplitudes (see Figure 9). The intercepts and slopes of the regressions were used for further statistical analyses. 


\section{Results}

Intercepts. Individual intercepts were entered into an ANOVA including the within-participant variables pressure and stimulus type. As expected, there was no significant effect (all $p \mathrm{~s}>.10$ ).

Slopes. Individual slopes were entered into an ANOVA including the within-participants variables pressure and stimulus type. There were significant main effects of the variables pressure $[F(1,7)=45.6, p<.001]$ and stimulus type $[F(2,14)=7.3, p<.05]$ and a significant interaction $[F(2,14)=6.5, p<.05]$. This pattern of results suggests that pressure did influence the slopes, but it influenced them differently for the different stimulus types. Hence, we analyzed the pressure effects on slope separately for each stimulus type. Slopes were significantly higher in the higher-pressure than in the lower-pressure condition for the force-signal bumps $[t(7)=5.5, p<.01]$ and the consistent bumps $[t(7)=3.6, p<.01]$, but not for the position-signal bumps $(p \mathrm{~s}>.5)$.

\section{Discussion}

Our results confirmed what we had expected. In conditions in which we varied the force-signal amplitudes of the stimuli (i.e., if force-signal or consistent bumps were presented), perceived amplitude increased more strongly with increasing (physical) stimulus amplitude if participants applied higher rather than lower pressure. In contrast, pressure did not alter the increase in perceived amplitude when only the position signal's amplitude varied. We argued above that the gain in the (absolutely) perceived amplitude derived from a single signal depends on both the signal-specific estimate of amplitude and the estimate's weight in the overall percept (remember that in the present experiment, the other signal is set to a constant value of 0 but is not strictly eliminated). Given the lack of a pressure effect for position-signal bumps, we conclude that pressure influenced neither the estimates derived from the position signal nor these estimates' weights in the percept. Because the estimate weights sum to 1 (see Equation 1), if the position-signal estimate's weight did not shift, the force-signal estimate's weight also did not shift. As a consequence, the pressure effects for force-signal bumps indicate that amplitude estimates derived from the same forcesignal amplitudes were larger for higher than for lower pressure. In the General Discussion, we discuss why only the force-signal estimate, not the position-signal estimate, may depend on the exploratory pressure.

Also note that participants were trained to match the haptic with the visual stimuli, but that the visually matched amplitudes still differed slightly from the physical (haptic) amplitudes. That is, participants judged the small stimuli as being larger than the corresponding physical ones, whereas they underestimated the large ones. However, in this experiment, we were not primarily interested in the exact (absolute) value of the perceived amplitudes; our conclusion is based only on the differences between perceived amplitudes in the different pressure conditions.

\section{GENERAL DISCUSSION}

In this study, we experimentally investigated to what extent and how variations in the pressure (and velocity) of exploratory movements influence the integration of force and position signals to haptically perceived bumps. The results from Experiment 1 demonstrate that the contribution of force signals to the overall bump percept increases with increasing pressure and decreases with increasing velocity. In addition, the reliability of the bump percept increases with higher pressure. Data from Experiment 2 further suggest that higher exploratory pressure particularly increases the reliability of the force signal to bumps, whereas it leaves the reliability of the position signal mostly unaffected. We applied the MLE model of optimal signal integration to the single-signal reliabilities to predict the reliability and the force-signal weight in the overall bump perception. These quantitative analyses predicted a pressure-related increase in the overall perceptual reliability. These predictions were closer to the measured values if the force and position signals were assumed to be correlated. However, shifts in the force-signal

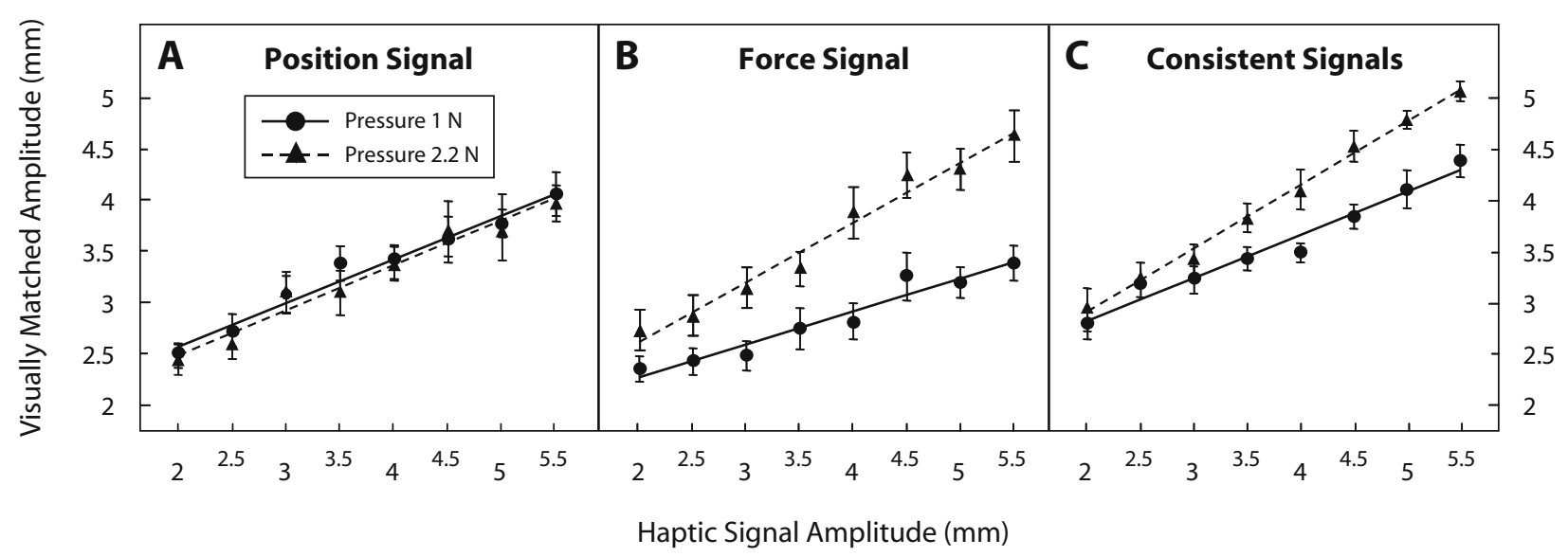

Figure 9. Experiment 3. Visually matched amplitude in the 1-N condition (solid lines) and the 2.2-N condition (dashed lines) for the position-signal bumps (A), force-signal bumps (B), and consistent bumps (C). Error bars are standard errors. 
weights could not be explained by variation in reliabilitydependent signal estimate weighting. The data from Experiment 3 suggest that the perceived amplitude of bumps, as well as the amplitude estimate derived from the forcesignal estimate, increases with increasing pressure. Note that this pressure-related bias in the force-signal estimate could explain the shift in force-signal weights.

\section{Why May the Force-Signal Estimate Vary With Pressure?}

Remember that the amplitude of a bump is perceived according to both force and position signals. The position signal is given by the shape of the finger trajectory during exploration. The shape of the trajectory does not vary with variation in the executed pressure. This is also what we found in Experiment 3: The perceived amplitude of the position-signal stimuli linearly increased with increasing position-signal amplitude $\left(A_{\mathrm{P}}\right)$, and this increase did not vary between the pressure conditions. But why did the absolute force-signal estimates increase with increasing pressure?

As described above, the force signal is defined by the directions of the reaction forces applied to the finger. If the brain's processing took into account the entire pattern of force direction change over time and space, it could recover the detailed shape from the force signalindependent of pressure or other exploratory parameters. However, our data show that the brain cannot use this invariant information to shape. A number of haptic illusions suggest that the interpretation of complicated force patterns can be erroneous (Roland, 1978; Rymer \& D'Almeida, 1980). Hence, we speculate that the brain can process only reduced aspects of the force signal (cf. Drewing \& Kaim, 2009) — aspects that vary with pressure. A good candidate for such a reduced aspect is the force component that horizontally decelerates and accelerates an exploratory finger movement (here, approximately the horizontal component). As schematically demonstrated in Figure 10, the magnitude of the horizontal force not only depends on a bump's slope (or its amplitude), but also increases with increasing pressure: For the same pressure, the horizontal component $F_{x}$ of the (normal) reaction force $F_{\mathrm{n}}$ is larger for the larger slope (B) than for the smaller slope (A), and is larger for the higher pressure (C) than for the lower pressure (A) when the slope is the same. In our experiments, the length of the bumps was constant. Thus, the slope linearly increased with the amplitude.

We were interested in whether variation in the perceived amplitude of force-signal bumps (i.e., stimuli with $A_{\mathrm{P}}=0$; see Figure 1C), measured in Experiment 3, might indeed be traced back to variation in the horizontal force component $F_{x}$. Therefore, we analyzed the horizontal forces measured by the PHANToM in the two pressure conditions. As shown in Figure 11, the horizontal force systematically varies during bump exploration. The magnitude of this variation increases with increasing amplitude, and it is higher when applying higher, as compared with lower, pressure. Figure 12 shows how the perceived amplitudes of the force-signal bumps (A) and the consistent bumps (B) relate to the magnitude of horizontal force variation in the lower-pressure condition (dotted lines) and the higher-pressure condition (dashed lines). For the force-signal bumps, linear regressions reveal essentially the same regression line ( 1 vs. $2.2 \mathrm{~N}$ : slope, 1.5 vs. 1.5 ; intercept, 1.7 vs. $1.5 \mathrm{~mm} ; r^{2}, .95$ vs. .99 ), indicating that variation in the horizontal force component alone predicts the perceived amplitude well. In other words, for the force-signal bumps, participants seem to interpret an increase in the horizontal force as higher amplitude, independent of whether this increase is caused by higher amplitude or by a higher applied pressure. In contrast, for the consistent bumps (Figure 12B), regression lines clearly differ between the lower- and higher-pressure conditions (slope, 2.3 vs. 2.0 ; intercept, 1.9 vs. $1.5 \mathrm{~mm} ; r^{2}, .97$ vs. $.99)$, indicating the additional contribution of the position signal. For example, a horizontal force component variation of $\sim 0.7 \mathrm{~N}$ was measured, on the one hand, for a consistent bump of $3.5 / 3.5\left(A_{\mathrm{P}} / A_{\mathrm{F}}\right)$ in the $1-\mathrm{N}$ condi-

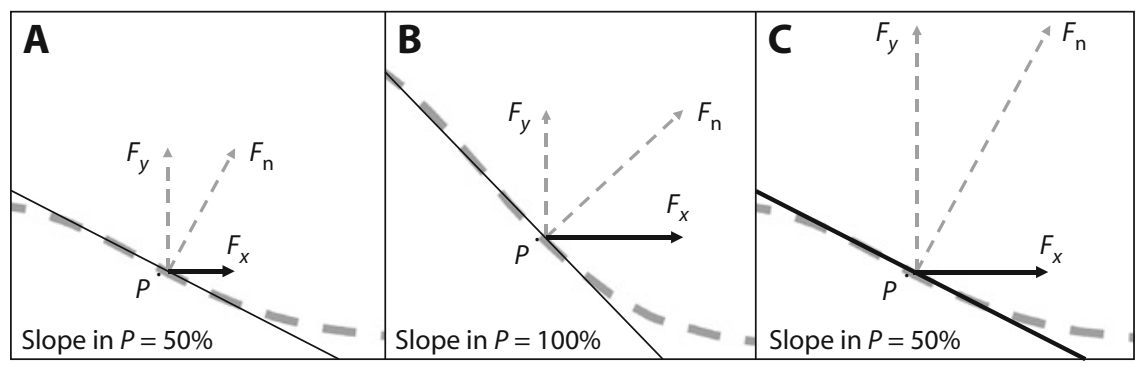

Pressure $=1 \mathrm{~N}$

Pressure $=1 \mathrm{~N}$

Pressure $=2.2 \mathrm{~N}$

Figure 10. Example of reaction forces acting on the finger during bump exploration at a single point $P$ (the finger moves from right to left). The dashed gray line illustrates the explored shape. The magnitude of the horizontal force component $F_{x}$ depends on both the magnitude and direction of the reaction force $F_{\mathrm{n}}$. Because, on the one hand, the direction of $F_{\mathrm{n}}$ depends on the slope, the magnitude of $F_{x}$ is larger for a higher slope (B) than for a lower slope (A). On the other hand, for the same slope, the magnitude of $F_{x}$ is larger for higher pressure $(C)$ than for lower pressure (A), because the magnitude of $F_{x}$ also depends on the magnitude of $F_{n}$, which in turn increases with increasing pressure. 


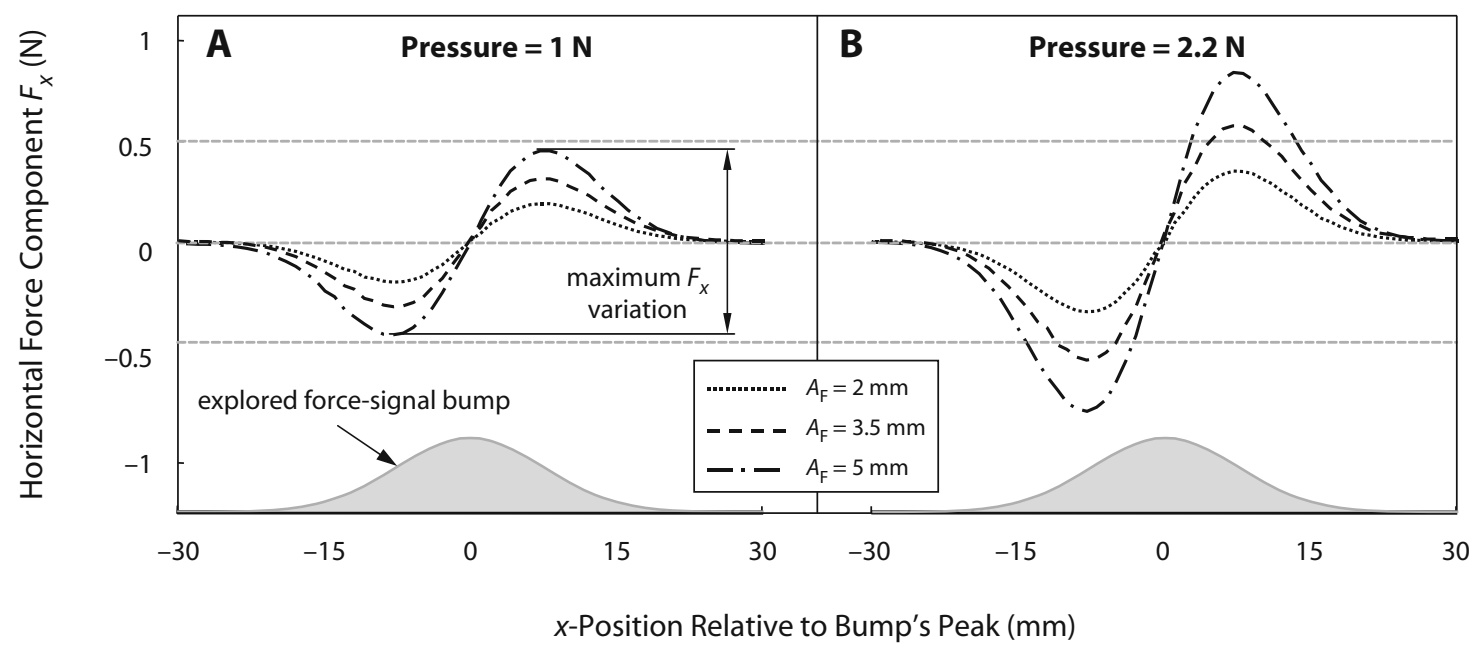

Figure 11. Average variation (over trials and participants) in the horizontal force component $F_{x}$ during the exploration of force-signal bumps with amplitudes of 2,3.5, and $5 \mathrm{~mm}$ in the 1-N (A) and 2.2-N (B) conditions (measure from a single trial). The finger moves from the right to the left. A force that decelerates the finger is defined as positive, and an accelerating force is negative.

tion. On the other hand, the same force variation $(\sim 0.7 \mathrm{~N})$ was measured for a 2.0/2.0 bump in the 2.2-N condition. Despite having the same variation in the horizontal force component, participants judged the $3.5 / 3.5$ bump as being clearly larger than the $2.0 / 2.0$ bump ( 3.45 vs. $2.84 \mathrm{~mm}$ ). This is easily explained by the contribution of the position signal to the overall amplitude estimate, because the position-signal amplitude was larger for the $3.5 / 3.5$ bump than for the 2.0/2.0 bump. Additionally, for the same lateral-force variation magnitudes, the perceived ampli- tudes of the consistent bumps (Figure 12B) were always larger than the perceived amplitudes of the force-signal bumps (Figure 12A), also indicating that both the force and position signals contributed to amplitude estimates for the consistent bumps.

Taken together, the integration of force and position signals into a shape percept, and in particular the influences of the exploratory movement parameters on this process, could not be fully explained by models of optimal integration. In particular, our results suggest that the absolute

\section{A Force-Signal Bumps}

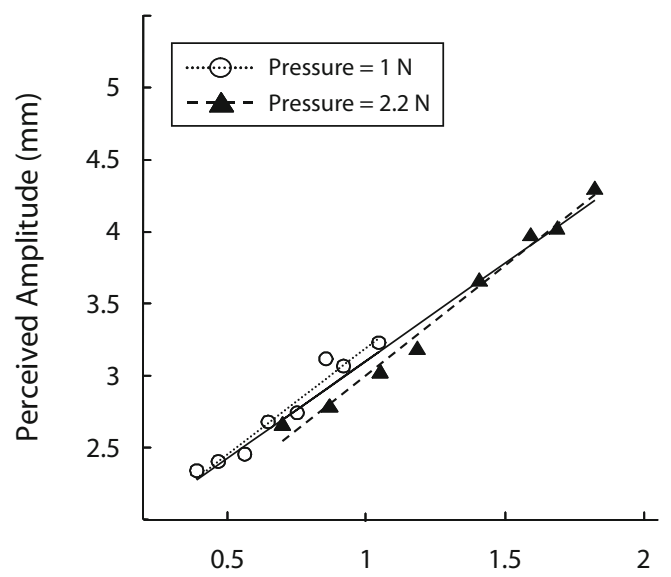

B Consistent Bumps

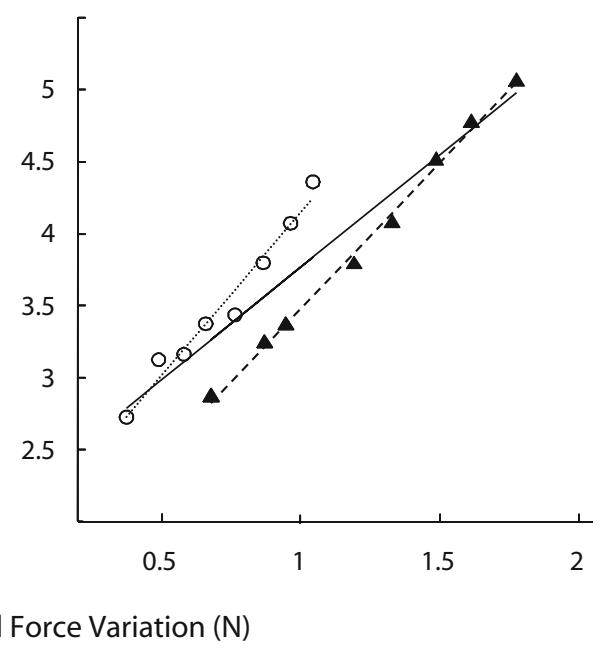

Figure 12. Average perceived amplitude of force-signal bumps (A) and consistent bumps (B) as a function of average maximum horizontal force variations in the lower-pressure condition (dotted lines) and the higher-pressure condition (dashed lines). Each symbol represents the values for a single (physical) stimulus amplitude. For the force-signal bumps, in which the position-signal amplitude is always 0 , slopes and intercepts of the regressions were nearly the same in the two pressure conditions, and linear regressions reveal essentially the same regression line (solid lines). For the consistent bumps, slopes and intercepts of the regressions clearly differed between pressure conditions. 
force-signal estimate increases with increasing exploratory pressure, because the brain uses only reduced aspects of the force signal. An influence of exploratory movement variation on the haptic percept has also been reported for other haptic modalities and exploratory procedures. For example, it has been shown that variation in exploratory behavior can differentially bias perceived weight (Flanagan \& Bandomir, 2000), extent (Wong, 1977), or roughness (Lederman, 1981; Lederman \& Taylor, 1972). The present study extends these findings, demonstrating that a haptically perceived shape can vary with variation in the executed pressure (i.e., the perceived amplitude increases with increasing pressure) and that this influence is due to pressure-related variation in the shape estimates derived from the force signals. This "illusion" should be considered when virtual haptic shapes are displayed by a force feedback device using position and force signals. One example is remote surgery: If a surgeon receives haptic feedback via a force feedback device about the shape of vitals being operated on, the shape of the vitals should not vary with the executed pressure. Another interesting finding in our study is that the relative contribution of a single signal to the overall percept seems to have increased with increasing magnitude of this signal relative to the magnitude of the other signal (Experiments 1 and 2). This effect also could not be explained by the MLE model, and it hints that further factors may also contribute to the integration process. The investigation of such factors beyond the MLE model poses interesting questions for future research on signal integration in active touch.

\section{AUTHOR NOTE}

This work was supported by a grant from the Deutsche Forschungsgemeinschaft (DR 730/1-1, 1-2; FOR 560). Thanks to Christoph Rasche and Kurt Debono for many helpful suggestions and stylistic advice. Correspondence concerning this article should be addressed to L. Kaim, Institute for Psychology, University of Giessen, Otto-Behaghel-Str. 10, 35394 Giessen, Germany (e-mail: lukas.kaim@psychol.uni-giessen.de).

\section{REFERENCES}

AlAis, D., \& BurR, D. (2004). The ventriloquist effect results from nearoptimal bimodal integration. Current Biology, 14, 257-262.

Backus, B. T., Banks, M. S., van Ee, R., \& Crowell, J. A. (1999). Horizontal and vertical disparity, eye position, and stereoscopic slant perception. Vision Research, 39, 1143-1170.

Blakemore, S.-J., Wolpert, D. M., \& Frith, C. D. (1998). Central cancellation of self-produced tickle sensation. Nature Neuroscience, 1, 635-640.

Drewing, K., \& ERnst, M. O. (2006). Integration of force and position cues for shape perception through active touch. Brain Research, 1078, 92-100.

Drewing, K., \& KaIM, L. (2009). Haptic shape perception from force and position signals varies with exploratory movement direction and the exploring finger. Attention, Perception, \& Psychophysics, 71, 1174-1184.

Drewing, K.,Wiecki, T. V., \& ERnst, M. O. (2008). Material properties determine how force and position signals combine in haptic shape perception. Acta Psychologica, 128, 264-273.

ERnSt, M. O., \& BANKS, M. S. (2002). Humans integrate visual and haptic information in a statistically optimal fashion. Nature, 415, 429-433.

Flanagan, J. R., \& Bandomir, C. A. (2000). Coming to grips with weight perception: Effects of grasp configuration on perceived heaviness. Perception \& Psychophysics, 62, 1204-1219.

Gepshtein, S., Burge, J., Ernst, M. O., \& Banks, M. S. (2005). The combination of vision and touch depends on spatial proximity. Journal of Vision, 5, 1013-1023.

Gibson, J. J. (1962). Observations on active touch. Psychological Review, 69, 477-491.

Hillis, J. M., WatT, S. J., Landy, M. S., \& BanKs, M. S. (2004). Slant from texture and disparity cues: Optimal cue combination. Journal of Vision, 4, 967-992.

JACOBS, R. A. (2002). What determines visual cue reliability? Trends in Cognitive Sciences, 6, 345-350.

Katz, D. (1969). Der Aufbau der Tastwelt. Darmstadt: Wissenschaftliche Buchgesellschaft. (Original work published 1925)

Klatzky, R. L., Lederman, S. J., \& Reed, C. (1989). Haptic integration of object properties: Texture, hardness, and planar contour. Journal of Experimental Psychology: Human Perception \& Performance, $15,45-57$.

Landy, M. S., Maloney, L. T., Johnston, E. B., \& Young, M. (1995). Measurement and modeling of depth cue combination: In defense of weak fusion. Vision Research, 35, 389-412.

Lederman, S. J. (1981). The perception of surface roughness by active and passive touch. Bulletin of the Psychonomic Society, 18, 253255

Lederman, S. J., \& KLAtzky, R. L. (1987). Hand movements: A window into haptic object recognition. Cognitive Psychology, 19, 342-368.

Lederman, S. J., \& TAYLOR, M. M. (1972). Fingertip force, surface geometry, and the perception of roughness by active touch. Perception \& Psychophysics, 12, 401-408.

O'Malley, M., \& GoldFarb, M. (2002). The effect of force saturation on the haptic perception of detail. IEEE/ASME Transactions on Mechatronics, 7, 280-288.

Oruç, I., Maloney, L. T., \& Landy, M. S. (2003). Weighted linear cue combination with possibly correlated error. Vision Research, $\mathbf{4 3}$, 2451-2468

Perotti, V. J., Todd, J. T., Lappin, J. S., \& Phillips, F. (1998). The perception of surface curvature from optical motion. Perception \& Psychophysics, 60, 377-388.

Robles-DE-LA-Torre, G., \& HaYward, V. (2001). Force can overcome object geometry in the perception of shape through active touch. $\mathrm{Na}$ ture, 412, 445-448.

RoLAND, P. E. (1978). Sensory feedback to the cerebral cortex during voluntary movement in man. Behavioral \& Brain Sciences, 1, 129-171.

Rosas, P., Wagemans, J., Ernst, M. O., \& Wichmann, F. A. (2005). Texture and haptic cues in slant discrimination: Reliability-based cue weighting without statistically optimal cue combination. Journal of the Optical Society of America A, 22, 801-809.

Rymer, W. Z., \& D'Almeida, A. (1980). Joint position sense: The effects of muscle contraction. Brain, 103, 1-22.

Srinivasan, M. A., \& LaMotte, R. H. (1995). Tactual discrimination of softness. Journal of Neurophysiology, 73, 88-101.

Vitello, M. P., ERnst, M. O., \& Fritschi, M. (2006). An instance of tactile suppression: Active exploration impairs tactile sensitivity for the direction of lateral movement. In Proceedings of EuroHaptics 2006 (pp. 351-355). Available at http://lsc.univ-evry.fr/ eurohaptics/ upload/cd/.

Voisin, J., Lamarre, Y., \& Chapman, C. E. (2002). Haptic discrimination of object shape in humans: Contribution of cutaneous and proprioceptive inputs. Experimental Brain Research, 145, 251-260.

WichmanN, F. A., \& Hill, N. J. (2001). The psychometric function: I. Fitting, sampling, and goodness of fit. Perception \& Psychophysics, 63, 1293-1313.

Wong, T. S. (1977). Dynamic properties of radial and tangential movements as determinants of the haptic horizontal-vertical illusion with an "L" figure. Journal of Experimental Psychology: Human Perception \& Performance, 3, 151-164.

Young, M. J., Landy, M. S., \& Maloney, L. T. (1993). A perturbation analysis of depth perception from combinations of texture and motion cues. Vision Research, 33, 2685-2696. 


\section{APPENDIX}

The correction function $T_{\mathrm{C}}\left(V, m_{j}\right)$ was defined as follows:

$$
T_{\mathrm{C}}\left(V, m_{j}\right)=a_{\mathrm{V}} a_{m j} \Delta A+\Delta m_{j}\left(a_{\mathrm{V}}-1\right),
$$

where the variables have the following meanings.

$a_{\mathrm{V}}$ : "Velocity factor"; increases the PHANToM reaction force for higher target velocity $V$ :

$$
a_{\mathrm{V}}=\frac{V}{0.3}+0.2
$$

$a_{m_{j}}$ : "Slope factor"; increases the reaction force when the finger rises, and decreases the force when the finger lowers ( $m_{j}=$ bump's slope at the current finger position):

$$
a_{m_{j}}=-0.8 \cdot m_{j} \text {. }
$$

$\Delta A$ : "Amplitude difference factor"; becomes larger, the larger force-signal amplitude $A_{\mathrm{F}}$ becomes relative to position-signal amplitude $A_{\mathrm{P}}$ :

$$
\Delta A=\frac{A_{\mathrm{F}}+20}{A_{\mathrm{P}}+20} .
$$

$\Delta m_{j}$ : "Slope variation factor"; increases the reaction force as the difference between the slope at the previous control cycle of the PHANToM $\left(m_{j-1}\right)$ and the slope at the current finger position $\left(m_{j}\right)$ becomes larger:

$$
\Delta m_{j}=4 \cdot\left(m_{j-1}-m_{j}\right)
$$

All numeric values were adjusted in an empirical manner. The correction term was developed especially for the Gaussian bumps we used, and it could not be used to improve the modulation of other shapes. Furthermore, this term requires that velocity and pressure remain constant during the exploratory stroke.

(Manuscript received February 9, 2009;

revision accepted for publication November 2, 2009.) 